\title{
The role of seven autoantibodies in lung cancer diagnosis
}

\author{
Bin Luo^, Guocai Mao, Haitao Ma, Shaomu Chen \\ Department of Thoracic Surgery, First Affiliated Hospital of Soochow University, Suzhou, China \\ Contributions: (I) Conception and design: B Luo; (II) Administrative support: S Chen, H Ma; (III) Provision of study materials or patients: S Chen, \\ G Mao; (IV) Collection and assembly of data: B Luo; (V) Data analysis and interpretation: B Luo; (VI) Manuscript writing: All authors; (VII) Final \\ approval of manuscript: All authors. \\ Correspondence to: Haitao Ma; Shaomu Chen. Department of Thoracic Surgery, First Affiliated Hospital of Soochow University, Suzhou, China. \\ Email: mht7403@163.com; michaelchensm@163.com.
}

Background: To investigate the expression and diagnostic value of seven autoantibodies (P53, PGP9.5, SOX2, GAGE7, GBU4-5, MAGE, and CACE) in lung cancer patients.

Methods: A total of 370 patients were admitted to the Thoracic Surgery of the First Affiliated Hospital of Suzhou University from 2017 to 2019, including 305 patients with lung cancer and 65 patients with benign lesions. The concentrations of the seven autoantibodies were determined by enzyme linked immunosorbent assay (ELISA). The expression levels of each antibody were compared between the two groups, and the levels of each antibody between lung cancer patients with different pathological types were also compared. We aimed to analyze the diagnostic efficiency of single antibody detection combined with seven antibodies, and also to explore whether there were differences among the positive rates of each antibody in sex, age, smoking history, pathological classification, and clinical stages in the lung cancer group.

Results: The expression levels of seven autoantibodies in the lung cancer group were higher than those in the benign lesion group. In the lung cancer group, the expression levels of the seven autoantibodies did not vary statistically among different pathological types. The area under the curve of combined detection of the seven antibodies reached 0.735 , and the Y-index reached 0.35 , which was higher than that of single antibody detection. P53 exhibited the highest sensitivity and lowest specificity; meanwhile, PGP9.5, SOX2, GAGE7, GBU4-5, and MAGEA1 exhibited low sensitivity and high specificity. The sensitivity and specificity of the CAGE were approximately $60 \%$, respectively. There was no statistical difference in the positive rate of each antibody in age, smoking history, and clinical stage. The positive rate of MAGEA1 and CAGE was statistically different in sex, and the positive rate of MAGEA1 was statistically different in pathological classification.

Conclusions: The seven autoantibodies of lung cancer can potentially be used as an auxiliary examination method for the early diagnosis of lung cancer.

Keywords: Lung cancer; seven autoantibodies; diagnosis

Submitted Apr 14, 2021. Accepted for publication Jun 08, 2021.

doi: $10.21037 /$ jtd-21-835

View this article at: https://dx.doi.org/10.21037/jtd-21-835

\section{Introduction}

Lung cancer is the most common malignant tumor in the world, which seriously threatens the health of people health. It the highest global age-standardized incidence rate (ASIR) and age-standardized mortality rate (ASMR) according to the Global Cancer Report 2016, with 2.1 million new cases and 1.8 million deaths occurring annually (1). The overall 5 -year survival rate for lung cancer is only $18.6 \%$, which is

^ ORCID: 0000-0001-9385-7932. 
primarily due to the fact that most patients are diagnosed at a late stage and miss the optimal time for treatment.

At present, screening methods for early lung cancer detection include low dose spiral CT (LDCT) and the serum tumor biomarkers method. The most widely used method is LDCT screening, which has high sensitivity (93.8\%). However, this method has low specificity and a high false positive rate, which leads to excessive medical treatment. Furthermore, its price and radiation damage may prevent it from becoming a routine method for early lung cancer screening (2). According to the DANTE tests conducted in Europe (3), for early-stage lung cancer patients, LDCT may facilitate a significantly lower-than-expected mortality rate. In recent years, the detection of autoantibodies in blood molecular markers has gradually revealed its value for early lung cancer screening. Autoantibody detection combined with CT examination can increase the specificity to $95.8 \%$, which greatly reduces the false positive rate, highlighting the potential of autoantibodies as an auxiliary examination for early diagnosis (4). Seven serum autoantibodies (P53, PGP9.5, SOX2, GAGE7, GBU4-5, MAGE, and CACE) were detected in this experiment to verify the value of autoantibodies in early lung cancer diagnosis, and to explore its potential use as an auxiliary method of early lung cancer diagnosis. Also, we tried to figure it out whether seven autoantibodies can be a method for accuracy diagnosis. In different pathological classification of lung cancer, some autoantibodies may can play an important role in distinguish. On the other hand, some autoantibodies have shown the potential to bet therapeutic targets.

We present the following article in accordance with the STARD reporting checklist (available at https://dx.doi. org/10.21037/jtd-21-835).

\section{Methods}

\section{General data}

A total of 370 patients admitted to the Department of Thoracic Surgery at the First Affiliated Hospital of Suzhou University from 2017 to 2019 were included in this study. All subjects were diagnosed with pulmonary nodule that could be a malignant one and received surgical treatment. Healthy people were not our projects According to the routine pathology results, 305 patients were diagnosed with lung cancer, while 65 patients were benign lesions patients. Of the 370 patients, 153 were men and 217 were women. Also,
162 subjects were over 60 years of age, and 208 subjects were under the age of 60 . There were 63 smokers, while the remaining 307 subjects had no smoking history. Of the 305 lung cancer patients, 267 were diagnosed with adenocarcinoma, including 97 adenocarcinomas in situ, three minimally invasive adenocarcinomas, and 167 invasive pulmonary adenocarcinomas. Moreover, there were 23 squamous cell carcinoma patients, 5 complex cancers, 5 large cell carcinomas, and 4 small cell carcinomas. TNM staging: I period in 285 cases, II period in 17 cases, III period in 1 case, and IV period in 2 cases.

\section{Inclusion/exclusion criteria}

Patients with lung nodules detected by high-resolution chest CT, which were highly suspected of being lung cancer based on clinical experience, were eligible for inclusion in this study. The exclusion criteria were as follows: (I) patients with distant metastasis after PET-CT detection; (II) those unable to accept surgery; (III) those who refused to accept serological detection of autoantibodies to lung cancer; and (IV) patients lacking clear pathological types and clinical TNM stages.

\section{Collections and processing}

Serum from $5 \mathrm{~mL}$ fasting blood was collected in the normal serum tube. The serum was separated after centrifugation and completed within 8 hours. If more than 8 hours passed, the serum should be stored at $2-8{ }^{\circ} \mathrm{C}$. The concentrations of the seven autoantibodies (P53, PGP9.5, SOX2, GAGE7, GBU4-5, MAGEA1, and CAGE) were determined by enzyme linked immunosorbent assay (ELISA). The autoantibody detection kit was purchased from Hangzhou Kai Paul Biotechnology Co., Ltd.

\section{The positive criteria}

The positive criteria for individual autoantibodies and the seven autoantibodies combined were $\mathrm{P} 53 \geq 13.1 \mathrm{U} / \mathrm{mL}$, PGP5.3 $\geq 11.1 \mathrm{U} / \mathrm{mL}$, SOX2 $\geq 10.3 \mathrm{U} / \mathrm{mL}$, GAGE7 $\geq 14.4 \mathrm{U} / \mathrm{mL}$, GBU4-5 $\geq 7.0 \mathrm{U} / \mathrm{mL}$, MAGE $\geq 11.9 \mathrm{U} / \mathrm{mL}$, CAGE $\geq 7.2 \mathrm{U} / \mathrm{mL}$ according to the reference values provided by the manufacturer. The positive criterion of the combined test as that if any of the seven autoantibodies was positive, then the combined test can be considered positive. However, the combined test results were negative when all seven autoantibodies were negative. 
Table 1 Expression of 7 autoantibodies in lung cancer group and benign lesion group

\begin{tabular}{lcccc}
\hline Antibody & Lung cancer group & Benign lesion group & $\mathrm{Z}$ & $\mathrm{P}$ \\
\hline P53 & $2.8(0.7,16.3)$ & $1.2(0.3,14.6)$ & -2.456 & 0.014 \\
PGP9.5 & $0.5(0.2,1.4)$ & $0.4(0.1,0.8)$ & -2.437 & 0.015 \\
SOX2 & $2.4(1.1,7.0)$ & $1.4(0.8,2.9)$ & -3.082 & 0.002 \\
GAGE7 & $2.2(1.0,5.4)$ & $1.7(0.6,2.7)$ & -2.747 & 0.006 \\
GBU4-5 & $0.8(0.3,3.9)$ & $0.5(0.2,1.4)$ & -2.133 & 0.033 \\
MAGEA1 & $0.4(0.2,1.6)$ & $0.3(0.1,0.7)$ & -2.863 & 0.004 \\
CAGE & $0.4(0.2,1.6)$ & $0.3(0.1,0.6)$ & -3.109 & 0.002 \\
\hline
\end{tabular}

\section{Statistical processing}

Statistical analysis was performed using SPSS24.0. All indicators were tested for normality using the Kolmogorov-Smirnov test, and the results showed that all of the indicators were in accordance with the non-normal distribution. Measurement data were expressed as median (Quartile) [M (p25, p75)]. The Mann-Whitney $\mathrm{U}$ test was used to compare the differences in expression levels between the two groups. The Kruskal-Wallis $\mathrm{H}$ test was used for intergroup comparisons; if the results exhibited differences, the Nemenyi test was used to further infer the difference between the two groups. The diagnostic efficacy of single autoantibody detection and combined detection was compared by constructing ROC (receiver operating characteristic) curves. Comparison of the positive rate between groups was performed using the chi-square test, corrected chi-square test, and Fisher exact probability method. $\mathrm{P}<0.05$ indicated a statistical difference, and $\mathrm{P}<0.01$ signified a significant statistical difference.

\section{Ethical statement}

The study was conducted in accordance with the Declaration of Helsinki (as revised in 2013). The study was approved by First Affiliated Hospital of Soochow University ethics and informed consent was taken from all the patients.

\section{Results}

\section{Comparison of autoantibodies between lung cancer group and benign lesions group}

The expression levels of the seven autoantibodies in the lung cancer group (P53, PGP9.5, SOX2, GAGE7, GBU4-5, MAGEA1, and CAGE) were higher than those of the benign lesions control group, and the results were statistically different $(\mathrm{P}<0.05)$. In the lung cancer group, the levels of SOX2, GAGE7, MAGEA1, and CAGE exhibited significant statistical differences $(\mathrm{P}<0.01)$. The specific values are shown in Table 1 .

\section{Comparison of the expression levels of the seven autoantibodies among different pathological types}

The 305 patients in lung cancer group were divided into the following groups according to their pathological types: an adenocarcinoma group [267], a squamous cell carcinoma group [23], and a non-adenocarcinoma non-squamous cell group [15]. The Kruskal-Wallis H tests results showed that all seven lung cancer autoantibodies were not statistically different in these three groups $(\mathrm{P}>0.05)$. The specific values are shown in Table 2.

Next, the adenocarcinoma group was further divided into adenocarcinoma in situ [97] and invasive pulmonary adenocarcinoma [167] groups. Since there were only three patients were diagnosed with minimally invasive adenocarcinoma, they were not included in the statistical analysis. Based on the Mann-Whitney $U$ test, seven lung cancer autoantibodies were not statistically different in the adenocarcinoma in situ and invasive pulmonary adenocarcinoma groups $(\mathrm{P}>0.05)$. The specific values are shown in Table 3.

\section{Evaluation of the diagnostic efficacy of autoantibodies in lung cancer detection}

The seven autoantibodies and combined tests were used as test variables. The sensitivity was ordinate, and reciprocal of the specificity was abscissa. We then 
Table 2 Expression of 7 autoantibodies in adenocarcinoma group, squamous cell carcinoma group and non-adenocarcinoma non-squamous cell group

\begin{tabular}{|c|c|c|c|c|c|}
\hline Antibody & Adenocarcinoma & Squamous cell carcinoma & Non-adenocarcinoma non-squamous cell & $\mathrm{H}$ & $\mathrm{P}$ \\
\hline PGP9.5 & $0.6(0.2,1.74)$ & $0.6(0.2,2.0)$ & $0.41(0.2,0.86)$ & 0.447 & 0.800 \\
\hline SOX2 & $2.4(1.1,5.8)$ & $2.4(1.1,15.1)$ & $3.9(1.1,13.8)$ & 1.273 & 0.529 \\
\hline GAGE7 & $2.2(0.9,5.8)$ & $1.68(1.05,4.9)$ & $2.5(1.13,5.7)$ & 0.44 & 0.802 \\
\hline MAGEA1 & $0.5(0.2,1.2)$ & $0.5(0.2,1.7)$ & $0.3(0.15,0.74)$ & 0.461 & 0.794 \\
\hline CAGE & $0.5(0.2,1.6)$ & $0.4(0.18,1.75)$ & $0.27(0.1,0.56)$ & 1.881 & 0.390 \\
\hline
\end{tabular}

Table 3 Expression of 7 autoantibodies in adenocarcinoma in situ group and invasive pulmonary adenocarcinoma group

\begin{tabular}{lccr}
\hline Antibody & Adenocarcinoma in situ & Invasive pulmonary adenocarcinoma & $Z$ \\
\hline P53 & $2.3(0.85,15.9)$ & $2.6(0.5,15.8)$ & -5.43 \\
PGP9.5 & $0.6(0.2,2.0)$ & $0.5(0.2,1.3)$ & -1.245 \\
SOX2 & $2.5(1.3,5.8)$ & $2.27(1.0,6.8)$ & -0.587 \\
GAGE7 & $2.2(0.9,6.9)$ & $2.2(0.94,5.0)$ & 0.213 \\
GBU4-5 & $1.3(0.4,4.9)$ & $0.72(0.3,3.4)$ & -0.453 \\
MAGEA1 & $0.5(0.2,1.3)$ & $0.5(0.2,1.2)$ & -1.6 \\
CAGE & $0.5(0.2,1.6)$ & $0.4(0.2,1.6)$ & -0.626 \\
\hline
\end{tabular}

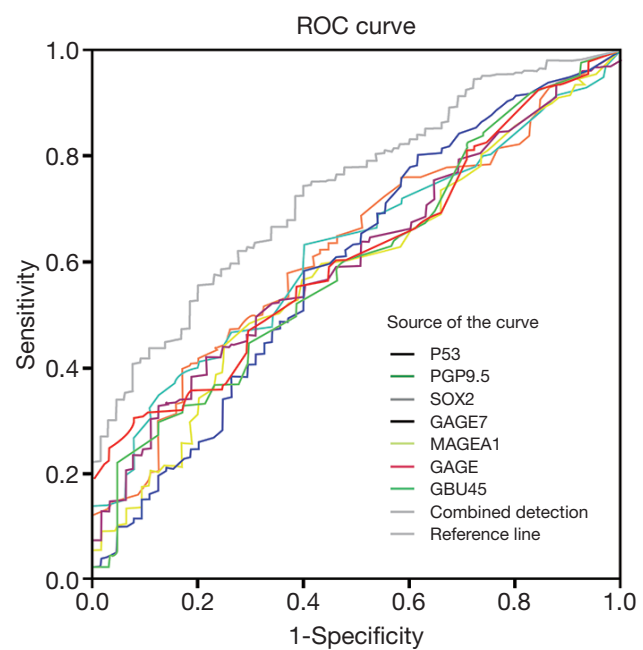

Figure 1 The receiver operating characteristic (ROC) curve analysis of seven autoantibodies and combined test in lung cancer.

constructed ROC curves to analyze the diagnostic efficacy of autoantibodies in lung cancer. We observed that
P53 had the highest sensitivity (80.3\%) and the lowest specificity (32\%). PGP9.5, SOX2, GAGE7, GBU4-5, and MAGEA1 exhibited higher specificity, however their sensitivity was relatively poor. The sensitivity (63.3\%) and specificity $(60 \%)$ of CAGE were almost equal. The seven autoantibodies showed $55.4 \%$ flexibility, and the specificity as $80 \%$. The AUC reached 0.735 (95\% CI: $0.674-0.795)$, and the Youden index (0.35) was also larger than that of the seven autoantibodies. According to the data analysis, P53 exhibited a unique advantage in diagnosing lung cancer, even higher than the combined detection. Compared with single autoantibody detection, the combined test had the largest AUC and Youden index, as well as better diagnostic efficacy. The specific values are shown in Figure 1 and Table 4.

\section{Comparison of the positive rates of the seven autoantibodies among different pathological types of lung cancer}

To analyze whether there were statistical differences in 
Table 4 Diagnostic efficacy of single autoantibody detection and combined test of seven autoantibodies

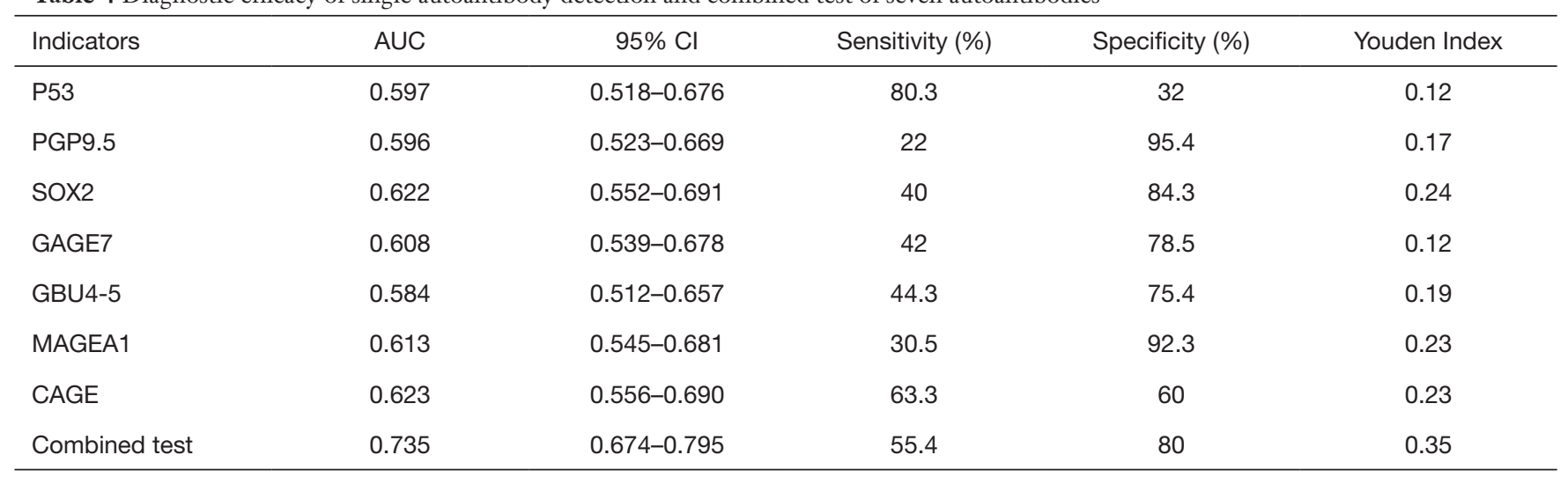

Table 5 Sex characteristics of lung cancer patients

\begin{tabular}{|c|c|c|c|c|c|c|c|c|c|c|c|c|c|c|}
\hline Groups & \multicolumn{2}{|c|}{ P53 } & \multicolumn{2}{|c|}{ PGP9.5 } & \multicolumn{2}{|c|}{ SOX2 } & \multicolumn{2}{|c|}{ GAGE7 } & \multicolumn{2}{|c|}{ GBU4-5 } & \multicolumn{2}{|c|}{ MAGEA1 } & \multicolumn{2}{|c|}{ CAGE } \\
\hline Male & 43 & 79 & 5 & 117 & 27 & 95 & 15 & 107 & 23 & 99 & 12 & 110 & 19 & 103 \\
\hline Female & 65 & 118 & 7 & 176 & 29 & 154 & 18 & 165 & 32 & 151 & 2 & 181 & 12 & 171 \\
\hline$\chi^{2}$ & \multicolumn{2}{|c|}{0.002} & \multicolumn{2}{|c|}{0.014} & \multicolumn{2}{|c|}{1.928} & \multicolumn{2}{|c|}{0.459} & \multicolumn{2}{|c|}{0.092} & \multicolumn{2}{|c|}{12.777} & \multicolumn{2}{|c|}{6.517} \\
\hline
\end{tabular}

Table 6 Age characteristics of lung cancer patients

\begin{tabular}{|c|c|c|c|c|c|c|c|c|c|c|c|c|c|c|}
\hline Groups & \multicolumn{2}{|c|}{ P53 } & \multicolumn{2}{|c|}{ PGP9.5 } & \multicolumn{2}{|c|}{ sox2 } & \multicolumn{2}{|c|}{ GAGE7 } & \multicolumn{2}{|c|}{ GBU4-5 } & \multicolumn{2}{|c|}{ MAGEA1 } & \multicolumn{2}{|c|}{ CAGE } \\
\hline$\geq 60$ & 52 & 86 & 5 & 117 & 27 & 95 & 15 & 107 & 23 & 99 & 12 & 110 & 19 & 103 \\
\hline$<60$ & 56 & 111 & 7 & 176 & 29 & 154 & 18 & 165 & 32 & 151 & 2 & 181 & 12 & 171 \\
\hline$\chi^{2}$ & \multicolumn{2}{|c|}{0.568} & \multicolumn{2}{|c|}{0.864} & \multicolumn{2}{|c|}{2.831} & \multicolumn{2}{|c|}{0.001} & \multicolumn{2}{|c|}{0.111} & \multicolumn{2}{|c|}{0.838} & \multicolumn{2}{|c|}{0.595} \\
\hline
\end{tabular}

the positive rates of autoantibodies in different groups, we divided the 305 lung cancer patients according to sex, age, smoking history, TNM stages, and pathological types. There 122 were male patients and 183 females; 138 subjects were over 60 years of age and 167 under 60 years of age; there were 52 smokers, while 253 subjects did not have a history of smoking; and TNM staging was as follows: I period in 285 cases, II period in 17 cases, III period in one case, and IV period in two cases.

Of the 305 lung cancer patients, there were 267 adenocarcinoma patients, 23 squamous cell carcinoma patients, and 15 non-adenocarcinoma non-squamous carcinoma patients. According to the statistical results, there were no significant differences in the positive rates of each antibody in age, smoking history, and clinical stage $(\mathrm{P}<0.05)$. The positive rates of MAGEA1 and CAGE were statistically different in sex. The MAGEA1 positive rate was statistically different in pathological classification. The specific values are shown in Tables 5-9.

\section{Discussion}

Lung cancer has the highest morbidity and mortality rates of any malignancy both in China and worldwide. Patients 
Table 7 Smoking history characteristics of lung cancer patients

\begin{tabular}{|c|c|c|c|c|c|c|c|c|c|c|c|c|c|c|}
\hline Smoking history & \multicolumn{2}{|c|}{ P53 } & \multicolumn{2}{|c|}{ PGP9.5 } & \multicolumn{2}{|c|}{ SOX2 } & \multicolumn{2}{|c|}{ GAGE7 } & \multicolumn{2}{|c|}{ GBU4-5 } & \multicolumn{2}{|c|}{ MAGEA1 } & \multicolumn{2}{|c|}{ CAGE } \\
\hline Yes & 21 & 31 & 2 & 50 & 12 & 40 & 6 & 46 & 11 & 41 & 5 & 47 & 7 & 45 \\
\hline No & 87 & 166 & 10 & 243 & 44 & 209 & 27 & 226 & 44 & 209 & 9 & 244 & 24 & 229 \\
\hline$\chi^{2}$ & \multicolumn{2}{|c|}{0.678} & \multicolumn{2}{|c|}{0.001} & \multicolumn{2}{|c|}{0.930} & \multicolumn{2}{|c|}{0.034} & \multicolumn{2}{|c|}{0.413} & \multicolumn{2}{|c|}{2.364} & \multicolumn{2}{|c|}{0.747} \\
\hline
\end{tabular}

Table 8 Clinical stage characteristics of lung cancer patients

\begin{tabular}{|c|c|c|c|c|c|c|c|c|c|c|c|c|c|c|}
\hline Clinical stage & \multicolumn{2}{|c|}{ P53 } & \multicolumn{2}{|c|}{ PGP9.5 } & \multicolumn{2}{|c|}{ SOX2 } & \multicolumn{2}{|c|}{ GAGE7 } & \multicolumn{2}{|c|}{ GBU4-5 } & \multicolumn{2}{|c|}{ MAGEA1 } & \multicolumn{2}{|c|}{ CAGE } \\
\hline I & 99 & 186 & 11 & 274 & 53 & 232 & 30 & 255 & 51 & 234 & 11 & 274 & 28 & 257 \\
\hline II + III + IV & 9 & 11 & 1 & 19 & 3 & 17 & 3 & 17 & 4 & 16 & 3 & 17 & 3 & 17 \\
\hline$\chi^{2}$ & \multicolumn{2}{|c|}{0.861} & \multicolumn{2}{|c|}{-} & \multicolumn{2}{|c|}{0.011} & \multicolumn{2}{|c|}{0.063} & \multicolumn{2}{|c|}{0.056} & \multicolumn{2}{|c|}{3.058} & \multicolumn{2}{|c|}{0.128} \\
\hline
\end{tabular}

Table 9 Pathological type characteristics of lung cancer patients

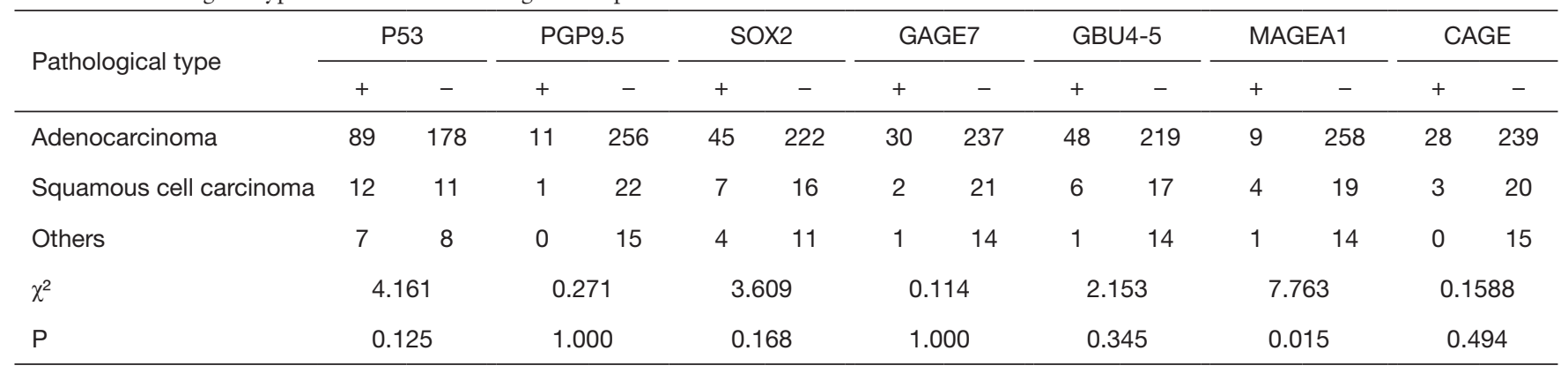

with early diagnosis have a higher 5-year survival rate. The 5-year survival rate of patients with non-small cell lung cancer is $92 \%$ in the IA stage and less than $10 \%$ in the IV stage (5). Therefore, early diagnosis is critical in the treatment of lung cancer. In the early stages of cancer, the body's immune system can recognize a small amount of abnormal proteins expressed by tumor cells through humoral immunity (i.e., tumor specific antigens), and produce antibodies against these antigens. Different kinds of lung cancer patients can detect tumor specific antigens in serum. These antibodies can be detected not only when lung cancer is diagnosed, but in some cases even 5 years before clinical symptoms appear (6). According to a study conducted by Chapman et al. (7), the sensitivity of the seven autoantibodies specific for Europeans (P53, c-myc, HER-2, NYESO1, CAGE, MUC1, and GBU4-5) is 76\% and the specificity is $92 \%$. The China Food and Drug Administration (CFDA) (8) specifically selected seven autoantibodies suitable for Chinese people.P53 can be detected in lung cancer patients as autoantibody produced by tumor suppressor genes. The MAGE-A1 belongs to the cancer/testicular antigen of the $\mathrm{X}$ chromosome cluster, expressed in a variety of tumors. GAGE is found in a variety of cancers, most common in melanoma, lung cancer. Expression of CAGE is associated with the cell cycle, and plays a role in cell growth and proliferation. GBU4-5, in the family of tumor-testicular products, is immunogenicity, cancer-specific, and may provide potential targets for 
the diagnosis and treatment of cancer.PGP9.5 increases deubiquitination of cyclin, which may lead to uncontrolled growth of tumor cells.SOX2 is expressed in lung cancer, breast cancer, ovarian cancer, and its ability to metastasize is proportional to its overexpression.

The gold standard for lung cancer is pathological diagnosis. According to the results of this study, the expression levels of the seven autoantibodies in the lung cancer group were higher than those in the benign lesion group $(\mathrm{P}<0.05)$, indicating that these seven autoantibodies can be used to distinguish lung cancer patients from benign lesion patients. They can be used as an auxiliary screening method for early lung cancer. Ren et al. (5) revealed that only PGP9.5, SOX2, GBU4-5, MAGEA1, and CAGE were found to have statistical differences between the lung cancer and benign lesion groups, while $\mathrm{P} 53(\mathrm{P}=0.371)$ and GAGE7 $(\mathrm{P}=0.957)$ were similar between the two groups. However, these results differ slightly from the results of our study, while the research conducted by $\mathrm{Mu}(9)$ was consistent with our experiment. According to the $2015 \mathrm{WHO}$ classification criteria for lung cancer (10), the lung cancer group was divided into an adenocarcinoma group, a squamous cell carcinoma group, and a non-adenocarcinoma non-squamous cell carcinoma group. Zhang et al. (11) found that the level of GBU4-5 in the adenocarcinoma group was higher than that in the squamous cell carcinoma group $(\mathrm{P}<0.05)$, while the expression of PGP9.5 in the adenocarcinoma group was higher than that in squamous cell carcinoma group. This suggests that GBU4-5 and PGP9.5 have the potential to distinguish adenocarcinomas from squamous cell carcinomas, although more research is required in the future. The adenocarcinoma group was further divided into an adenocarcinoma in situ group and an invasive pulmonary adenocarcinoma group. The expression of autoantibodies in these two groups was not statistically different and could not be used to distinguish specific adenocarcinoma types. This indicates that although autoantibodies can be used for early lung cancer screening, they cannot be used as a means of accurate diagnosis. This result is consistent with Wang et al. (8) conclusion.

According to the ROC curve of seven autoantibodies and combined detection, P53 exhibited the highest sensitivity and the lowest specificity, indicating that P53 had a high misdiagnosis rate. For the first time, Infante (12) found that P53 was associated with tumor progression, with a sensitivity of $27 \%$ and a specificity of $90 \%$. The reason for this is that P53 is expressed not only in lung cancer, but also in numerous other malignant tumors (including colorectal cancer, breast cancer, etc.). Parrales et al. (13) discovered that P53 is expressed in more than $50 \%$ of cancers, which is closely related to the activity of tumor cells and is a potential cancer therapeutic target. PGP9.5, SOX2, GAGE7, GBU4-5, and MAGEA1 exhibited low sensitivities and high specificities, indicating that these five antibodies had a higher misdiagnosis rate. However, the sensitivity and specificity of the CAGE were both approximately $60 \%$, which indicated that this antibody had a suitable misdiagnosis rate. Yeon et al. (14) reported that CAGE was related to the drug resistance of anticancer drugs. By combining with CAGE derivatives, it is hoped that overcoming the drug resistance of non-small cell lung cancer can be achieved. Combined autoantibody detection had a sensitivity of $55.4 \%$, a specificity of $80 \%$, an area under the curve of 0.735 , and a Youden index of 0.35 , which was higher than that of single antibody detection, indicating that combined detection has better diagnostic efficiency. This finding was consistent with the conclusion of $\mathrm{Du}$ et al. (4), who reported that combined detection of seven autoantibodies was more advantageous than single antibody detection. According to Ren et al. (5), the sensitivity of combined test was $61 \%$, specificity was $90 \%$, and area under the curve were 0.781 , which highlighted the superior diagnostic efficacy of combined detection. The meta-analysis conducted by Qin et al. (15) found the following: (I) GBU4-5 had a sensitivity of $7 \%$, a specificity of $98 \%$, and an area under the curve of 0.91 ; (II) CAGE had a sensitivity of $14 \%$, a specificity of $98 \%$, and an area under the curve of 0.90 ; and (III) SOX2 had a sensitivity of $14 \%$, a specificity of $99 \%$, and an area under the curve of 0.93 . These autoantibodies had high sensitivity, but low specificity. Combined detection can provide high specificity but low sensitivity.

We also compared the positive rates of seven autoantibodies in lung cancer patients with different clinicopathological characteristics. We observed that there were no statistical differences in age, smoking history, and clinical stage $(\mathrm{P}>0.05)$, while the positive rates of MAGEA1 and CAGE were statistically significant in sex. Also, the positive rate of MAGEA1 was statistically significant in pathological classification. The distribution of P53, PGP9.5, SOX2, GAGE7, and GBU4-5 was uniform in age, sex, smoking history, clinical stage, and pathological classification, which was consistent with the results Zhang et al. (11). According to Hibi et al. (16), PGP9.5 was associated with the clinical stage of lung cancer (the positive rate of lung cancer was $44 \%$ in stage 
I, and $75 \%$ in stages II and III stage). The meta-analysis by Li et al. (17) revealed that SOX2 was associated with sex and smoking history, and was highly expressed in squamous cell carcinomas. Moreover, the meta-analysis by Chen et al. (18) found that SOX2 had nothing to do with gender and smoking history. At the same time, it had high expression in squamous cell carcinomas, which indicated better prognosis in different pathological types of lung cancer, suggesting that SOX2 has the potential to become a prognostic indicator. Gjerstorff et al. (19) revealed that the GAGE7 was not related to the pathological classification of lung cancer, but its expression in stages II and IIIa was significantly higher than that in stage I. The positive rate of CAGE varies between different sexes, and these differences should be excluded when using CAGE to diagnose lung cancer patients. Also, the positive rate of MAGEA1 was different between different sexes and pathological types. This suggested that MAGEA1 had the potential to act as a precise diagnosis autoantibody. According to Sang et al. (20), the expression rate of MAGE-A in lung adenocarcinoma is $46.66 \%$, which was related to a lower 10 -year survival rate and was a marker of poor prognosis. MAGE-A may be used as a prognostic assessment factor and a potential therapeutic target.

All of the data included in this experiment were taken from the thoracic surgery database. Most of the patients had stage I adenocarcinoma, however there was a lack of patients with other pathological classifications and clinical stages, which resulted in discrepancies between some of our conclusions and those of other scholars. Thus, we need to strengthen cooperation with the respiratory and oncology departments in the future, in order to expand the scope and number of patients in future trials. This will increase the number of patients with different types, and facilitate more hierarchical research.

In summary, the value of autoantibodies as an auxiliary means of early lung cancer screening was confirmed. However, these autoantibodies did not exhibit obvious advantages for accurate diagnosis. The diagnostic efficacy of the combined detection of the seven autoantibodies was higher than that of single antibody detection, however the sensitivity and specificity were not as high as those of some single antibodies. Therefore, comprehensive evaluation is required for diagnosis.

\section{Acknowledgments}

Funding: None.

\section{Footnote}

Reporting Checklist: The authors have completed the STARD reporting checklist. Available at https://dx.doi. org/10.21037/jtd-21-835

Data Sharing Statement: Available at https://dx.doi. org/10.21037/jtd-21-835

Conflicts of Interest: All authors have completed the ICMJE uniform disclosure form (available at https://dx.doi. org/10.21037/jtd-21-835). The authors have no conflicts of interest to declare.

Ethical Statement: The authors are accountable for all aspects of the work in ensuring that questions related to the accuracy or integrity of any part of the work are appropriately investigated and resolved. The study was conducted in accordance with the Declaration of Helsinki (as revised in 2013). The study was approved by First Affiliated Hospital of Soochow University ethics and informed consent was taken from all the patients.

Open Access Statement: This is an Open Access article distributed in accordance with the Creative Commons Attribution-NonCommercial-NoDerivs 4.0 International License (CC BY-NC-ND 4.0), which permits the noncommercial replication and distribution of the article with the strict proviso that no changes or edits are made and the original work is properly cited (including links to both the formal publication through the relevant DOI and the license). See: https://creativecommons.org/licenses/by-nc-nd/4.0/.

\section{References}

1. Miller KD, Siegel RL, Lin CC, et al. Cancer treatment and survivorship statistics, 2016. CA Cancer J Clin 2016;66:271-89.

2. Boiselle PM. Computed tomography screening for lung cancer. Jama 2013;309:1163-70.

3. Li Y, Karjalainen A, Koskinen H, et al. p53 autoantibodies predict subsequent development of cancer. Int J Cancer 2005;114:157-60.

4. Du Q, Yu R, Wang H, et al. Significance of tumorassociated autoantibodies in the early diagnosis of lung cancer. Clin Respir J 2018;12: 2020-8.

5. Ren S, Zhang S, Jiang T, et al. Early detection of lung cancer by using an autoantibody panel in Chinese 
population. Oncoimmunology 2017;7:e1384108.

6. Chapman CJ, Healey GF, Murray A, et al. EarlyCDT®Lung test: improved clinical utility through additional autoantibody assays. Tumour Biol 2012;33:1319-26.

7. Chapman CJ, Murray A, McElveen JE, et al. Autoantibodies in lung cancer: possibilities for early detection and subsequent cure. Thorax 2008;63:228-33.

8. Wang $\mathrm{W}$, Zhuang $\mathrm{R}, \mathrm{Ma} \mathrm{H}$, et al. The diagnostic value of a seven-autoantibody panel and a nomogram with a scoring table for predicting the risk of non-small-cell lung cancer. Cancer Sci 2020;111:1699-710.

9. Mu Y, Xie F, Sun T. Clinical value of seven autoantibodies combined detection in the diagnosis of lung cancer. J Clin Lab Anal 2020;34:e23349.

10. Travis WD, Brambilla E, Nicholson AG, et al. The 2015 World Health Organization Classification of Lung Tumors: Impact of Genetic, Clinical and Radiologic Advances Since the 2004 Classification. J Thorac Oncol 2015;10:1243-60.

11. Zhang R, Ma L, Li W, et al. Diagnostic value of multiple tumor-associated autoantibodies in lung cancer. Onco Targets Ther 2019;12:457-69.

12. Infante M, Angeli E, Chiarenza M, et al. A randomized study of lung cancer screening with spiral computed tomography: three-year results from the DANTE trial. Am J Respir Crit Care Med 2009;180:445-53.

13. Parrales A, Iwakuma T. Targeting Oncogenic Mutant p53 for Cancer Therapy. Front Oncol 2015;5:288.

Cite this article as: Luo B, Mao G, Ma H, Chen S. The role of seven autoantibodies in lung cancer diagnosis. $\mathrm{J}$ Thorac Dis 2021;13(6):3660-3668. doi: 10.21037/jtd-21-835
14. Yeon M, Byun J, Kim H, et al. CAGE Binds to Beclin1, Regulates Autophagic Flux and CAGE-Derived Peptide Confers Sensitivity to Anti-cancer Drugs in Non-small Cell Lung Cancer Cells. Front Oncol 2018;8:599.

15. Qin J, Zeng J, Yang T, et al. Diagnostic Value of Autoantibodies in Lung Cancer: a Systematic Review and Meta-Analysis. Cell Physiol Biochem 2018;51:2631-46.

16. Hibi K, Westra WH, Borges M, et al. PGP9.5 as a candidate tumor marker for non-small-cell lung cancer. Am J Pathol 1999;155:711-5.

17. Li Q, Liu F, Zhang Y, et al. Association of SOX2 and Nestin DNA amplification and protein expression with clinical features and overall survival in non-small cell lung cancer: A systematic review and meta-analysis. Oncotarget 2016;7:34520-31.

18. Chen Y, Huang Y, Chen J, et al. The prognostic value of SOX2 expression in non-small cell lung cancer: a metaanalysis. PLoS One 2013;8:e71140.

19. Gjerstorff MF, Olsen KE, Ditzel HJ, et al. Analysis of GAGE, NY-ESO-1 and SP17 cancer/testis antigen expression in early stage non-small cell lung carcinoma. BMC Cancer 2013;13:466.

20. Sang M, Gu L, Yin D, et al. MAGE-A family expression is correlated with poor survival of patients with lung adenocarcinoma: a retrospective clinical study based on tissue microarray. J Clin Pathol,2017;70:533-40.

(English Language Editor: A. Kassem) 